\title{
OPÇÕES CONTRACEPTIVAS PARA REDUÇÃO DE EVENTOS TROMBOEMBÓLICOS EM MULHERES DIABÉTICAS
}

\author{
CONTRACEPTIVE OPTIONS FOR REDUCING THROMBOEMBOLIC \\ EVENTS IN DIABETIC WOMEN
}

\author{
Andressa Daiana Nascimento do Carmo ${ }^{1}$, Adroaldo Lunardelli ${ }^{2}$.
}

\section{G ACESSO LIVRE}

Citação: Carmo ADN, Lunardelli A (2019) Opções contraceptivas para redução de eventos tromboembólicos em mulheres diabéticas. Revista de Patologia do Tocantins, 6(1): 63-69.

\section{Instituição: $\quad$ BBiomédica. Centro} Universitário Ritter dos Reis (UniRitter). Porto Alegre, Rio Grande do Sul, Brasil.

${ }^{2}$ Farmacêutico Doutor. Professor do Centro Universitário Ritter dos Reis (UniRitter). Porto Alegre, Rio Grande do Sul, Brasil.

\section{Autor correspondente: Adroaldo Lunardelli; adroaldo_lunardelli@uniritter.edu.br}

Editor: Guedes V. R. Medicina, Universidade Federal do Tocantins, Brasil.

\section{Publicado: 06 de maio de 2019.}

Direitos Autorais: (c) 2019 Carmo et al. Este é um artigo de acesso aberto que permite o uso, a distribuição e a reprodução sem restrições em qualquer meio, desde que o autor original e a fonte sejam creditados.

Conflito de interesses: os autores declararam que não existem conflitos de interesses.

\begin{abstract}
RESUMO
Introdução: A diabetes mellitus é um grave e crescente problema de saúde pública, podendo levar à consequências irreversíveis na saúde de seus portadores, prevendo-se crescimento no número de novos portadores para os próximos anos. $O$ uso de métodos contraceptivos hormonais também cresceu nos últimos anos, e com o retardamento das gestações, estes números tendem a aumentar ainda mais. A prescrição de contracepção hormonal para mulheres diabéticas deve ser cuidadosamente avaliada, pois a presença da doença já confere risco para eventos adversos à saúde da paciente, em especial os eventos tromboembólicos. Objetivos: O estudo objetivou revisar a literatura quanto aos métodos contraceptivos hormonais mais adequados para pacientes diabéticos, buscando redução de eventos adversos e melhora na qualidade de vida destes.

Materiais e métodos: Trata-se de uma revisão integrativa da literatura onde foram acessadas as bases de dados Medline/Pubmed, ScienceDirect e Scielo. Utilizou-se os descritores contraceptive, thrombosis e diabetes, bem como a combinação destas palavras.

Resultados: Foram encontrados 4 trabalhos que se enquadraram na temática do estudo e foram incluídos na revisão. Apenas 1 foi realizado exclusivamente com pacientes diabéticas. Conclusão: A utilização de contracepção hormonal combinada apresenta a mesma probabilidade de causar eventos tromboembólicos em mulheres com e sem diabetes, mas o risco absoluto em mulheres com diabetes é maior devido à doença, que é um agravante para estes incidentes. mulheres diabéticas que utilizam contracepção com estrogênio têm risco maior de desenvolver complicações trombóticas quando comparadas às que utilizam progestogênio ou as não usuárias.

Palavras-chave: diabetes mellitus; anticoncepção; estrogênios; progesterona; tromboembolia.
\end{abstract}

\begin{abstract}
Introduction: Diabetes mellitus is a serious and growing public health problem, and may lead to irreversible consequences for the health of its patients, with a projected increase in the number of new patients for the coming years. The use of hormonal contraceptive methods has also grown in recent years, and with the delay of pregnancies, these numbers tend to increase even more. The prescription of hormonal contraception for diabetic women should be carefully evaluated, since the presence of the disease already confers risk to adverse events to the patient's health, especially thromboembolic events.

Objective: The present study aimed to review the literature regarding on the most appropriate hormonal contraceptive methods for diabetic patients, seeking to reduce adverse events and improve their quality's life.

Materials and Methods: It is an integrative literature review conducted by searching the databases Medline/Pubmed, ScienceDirect and Scielo. The following descriptors were used: contraceptive, thrombosis and diabetes, as well as the combination of these words.

Results: Four papers were found that fit the theme of the study and were included in the review. Only 1 was performed exclusively with diabetic patients.

Conclusion: Using combined hormonal contraception has the same probability of causing thromboembolic events in women with and without diabetes, but the absolute risk in women with diabetes is greater because of the disease, which is an aggravating factor for these incidents. Diabetic women who use estrogen contraception are at greater risk of developing thrombotic complications when compared to those using progestogen or non-users.
\end{abstract}

Keywords: diabetes mellitus; contraception; estrogens; progesterone; thromboembolism. 
INTRODUÇÃO

A contracepção não é um conceito dos dias atuais, há cerca de dois mil anos já eram utilizados métodos contraceptivos rudimentares para prevenir gestações e o infanticídio. $O$ primeiro contraceptivo hormonal foi lançado no mercado nos Estados Unidos, na década de 60, e continha a combinação de $10 \mu \mathrm{g}$ de progesterona (noretinodrel) e $150 \mu \mathrm{g}$ de estrogênio (mestranol) ${ }^{1}$. Os anticoncepcionais hormonais orais se subdividem em duas categorias: os anticoncepcionais orais combinados (AOC) e os anticoncepcionais orais apenas com progestinas (com doses mais baixas de hormônios em comparação com os AOCs). Os AOCs são compostos de um etinilestradiol (EE) e um progestágeno, podendo ser monofásico, difásico ou trifásico, e ainda podem se diferenciar de acordo com a sua geração, podendo ser de primeira, segunda ou terceira ${ }^{2}$.

A facilidade e simplicidade dos fármacos administrados por via oral tornam esta classe bastante popular, todavia, outras formas de contracepção podem ser encontradas no mercado. Os contraceptivos injetáveis também se subdividem em combinados (etinilestradiol com progestinas) e os que possuem apenas progestinas ${ }^{3}$. $O$ anel vaginal é composto por $15 \mu \mathrm{g}$ de EE e $120 \mu \mathrm{g}$ de etonogestrel diário, sendo instalado na vagina e liberando os hormônios no seu interior, atingindo níveis estáveis na circulação sanguínea a fim de inibir a ovulação ${ }^{4}$. A contracepção de emergência possui duas apresentações, a que é composta de estrogênio sintético e progesterona (conhecido como método YUSPE) e a opção que possui apenas progesterona isolada (levonogestrel) com dose total de $1,5 \mathrm{mg}^{5}$.

Outras opções contraceptivas são os métodos de longa duração (LARC). O implante subdérmico, que é composto apenas com progestinas, é implantado - geralmente - no braço e libera hormônio durante três anos. 0 implante duplo, onde se utiliza dois bastões e tem validade de cinco anos, já está aprovado nos Estados Unidos ${ }^{6}$. O dispositivo intrauterino (DIU) de levonogestrel é implantado no útero, mede cerca de $3,2 \mathrm{~cm}$ de comprimento e tem um reservatório de $52 \mathrm{mg}$ de levonogestreol que, através da sua membrana de controle, libera no plasma (após 15 minutos da inserção) entre 11 e 14 $\mu \mathrm{g}$ do hormônio por dia, até chegar a $11 \mu \mathrm{g} /$ dia no final da sua vida útil, que é de cinco anos ${ }^{7}$.

Existem vários fatores de risco para a utilização de métodos contraceptivos hormonais, dentre eles estão a hiperlipidemia, obesidade, pressão arterial aumentada, histórico de acidente vascular cerebral (AVC), histórico de infarto agudo do miocárdio (IAM), histórico de trombose venosa (TV) na família e a diabetes mellitus (DM) ${ }^{8}$. Quando a prescrição de métodos contraceptivos é feita para mulheres que são afetadas pelo DM tipo I ou II, deve-se ter um cuidado ainda mais especial. Mulheres que pertencem a este grupo possuem restrições quanto ao tipo de contraceptivo e quanto ao tipo de hormônio com que ele é feito. Estes métodos devem possuir uma eficácia elevada, para se prevenir gestações não planejadas aliado a um baixo índice de possíveis complicações causadas por hormônios. $O$ tipo de método utilizado neste grupo de mulheres requer uma maior atenção do profissional da saúde que a acompanha, buscando sempre a redução de possíveis complicações no quadro clínico destas pacientes ${ }^{8}$.
A diabetes mellitus (DM) é um distúrbio metabólico caracterizado pela incapacidade congênita ou adquirida de transportar açúcar da corrente sanguínea para as células ${ }^{9}$. É uma doença crônica caracterizada por altos níveis de glicose no sangue (glicemia) em jejum classificada em dois tipos: tipo I (um), caracterizado como diabetes mellitus insulinodependente, causada devido a incapacidade de secreção do hormônio insulina por parte do pâncreas; e tipo II (dois), diabetes mellitus não insulinodependente. Trata-se, este tipo, do mais comum, correspondendo a aproximadamente de 90-95\% do número total de casos e sua origem ocorre através da combinação entre resistência à ação da insulina e da resposta secretora compensatória inadequada deste hormônio. Pacientes portadores do diabetes mellitus tipo II evoluem, ao longo do tempo, para a diabetes mellitus tipo I. Isto se deve ao fato de que a constante resistência à insulina seja responsável por gerar a hiperinsulinemia, ou seja, no aumento da concentração desse hormônio no plasma. A falência das células beta pancreáticas no diabetes mellitus tipo II ocorre quando as mesmas se tornam incapazes de fazer a compensação da resistência à insulina. Esta falência é progressiva com perda de células beta, particularmente quando a hiperglicemia está estabelecida. Em seguida, há diminuição da secreção de insulina devido à progressiva redução da função das células beta (responsáveis pela produção da insulina), havendo hipossensibilidade destas células à glicose, fato este relacionado ao esgotamento funcional ocasionado pela hiperestimulação inicial ao avançar da idade do indivíduo acometido pela doença ${ }^{10}$.

Um dos agravos da associação entre diabetes mellitus e contraceptivos hormonais são os eventos tromboembólicos, tanto venosos quanto arteriais, já que o diabetes mellitus é um fator acelerador de doenças do sistema vascular, aumentando o risco de esta mulher desenvolver patologias que, muitas vezes, podem ser fatais. Complicações mais graves, como cardiovasculares, cerebrovasculares, renais e retinopatias, levam a um grande número de internações e elevam os gastos dos serviços de saúde, principalmente o SUS (sistema único de saúde), e também a óbito ${ }^{11}$. Com o aumento do número de casos de diabetes mellitus em todo mundo, incluído o Brasil ${ }^{12}$, e do impacto que esta doença pode causar na qualidade de vida da população, em especial para mulheres em idade reprodutiva, nota-se a importância da realização de uma revisão integrativa da literatura, afim de evidenciar os métodos contraceptivos hormonais mais adequados para este grupo de pacientes, buscando redução de eventos adversos e melhora na qualidade de vida destes.

\section{MATERIAS E MÉTODOS}

O presente estudo consiste em uma revisão integrativa da literatura com base na busca de artigos científicos disponíveis em diferentes bancos de dados. A busca foi realizada entre os meses de agosto a novembro de 2018, nas bases de dados Medline/Pubmed e Science Direct, com as seguintes combinações de palavras chaves em inglês: contraceptive, thrombosis e diabetes, sendo os mesmos temas pesquisados na base de dados Scielo para buscas em português (contraceptivo, trombose e diabetes mellitus).

Foram adotados como critério de inclusão as 
publicações dos últimos dez anos que abordassem temas relacionados com contraceptivos hormonais, dispositivo intrauterino (DIU), diabetes mellitus, tromboses (venosas e arteriais), infarto agudo do miocárdio e acidente vascular cerebral. Primeiramente, foram analisados títulos e resumos dos resultados das buscas, sendo selecionados aqueles estudos que contemplaram a temática proposta, incluídos e lidos de forma detalhada. Foram excluídos da seleção estudos realizados em pacientes não diabéticos e com animais. A análise seguiu referencial teórico, incluindo a leitura exploratória, seletiva, analítica e interpretativa do material obtido.

\section{RESULTADOS E DISCUSSÃO}

Através das análises feitas, seguindo os critérios de inclusão acima descritos, foram encontrados 139 artigos no total, sendo que, 115 foram excluídos por data, restando 22 artigos que foram analisados na íntegra. Destes, 18 foram excluídos por não se encaixarem na temática do estudo, pois abordavam tópicos (síndrome de ovários policísticos, mulheres obesas, ressecção intestinal, amputação de membros, estudos que não incluíam pacientes diabéticas e estudos que não foram realizados em humanos) alhures ao intento deste desígnio. A Figura 1 mostra a sequência de trabalhos encontrados e classificados. Quatro trabalhos, portanto, foram selecionados por serem considerados aptos para o presente estudo; eles estão apresentados na Tabela 1 . Os trabalhos incluídos no estudo falam sobre a relação entre a contracepção hormonal em mulheres de idade reprodutiva com diabetes mellitus tipo I e II e a associação desta doença de base com eventos tromboembólicos, cardiovasculares e cerebrovasculares. Estudos mostrando a relação entre contracepção e eventos tromboembólicos (tanto venosos quanto arteriais) são amplamente divulgados, mas estes estudos geralmente não incluem mulheres diabéticas, e quando às incluem, não as isolam, deixando este importante grupo da sociedade em uma lacuna que pode ser perigosa. Embora o risco de uma mulher saudável desenvolver tais eventos seja baixo, mulheres com diabetes mellitus tipo I e II têm risco aumentado ${ }^{13}$.

Tabela 1 - Trabalhos selecionados e contemplados no presente estudo com respectivos autores, títulos, ano e periódico de publicação.

\begin{tabular}{ccc}
\hline Autores & Título e ano & Periódico \\
\hline $\begin{array}{c}\text { O'Brien SH } \\
\text { et al. }{ }^{22}\end{array}$ & $\begin{array}{c}\text { Hormonal contraception and risk } \\
\text { of thromboembolism in women } \\
\text { with diabetes, } \\
2017 .\end{array}$ & Diabetes Care \\
\hline $\begin{array}{c}\text { Lidegaard } \\
\emptyset^{29}\end{array}$ & $\begin{array}{c}\text { Hormonal contraception, } \\
\text { thrombosis and age, } \\
\text { 2014. }\end{array}$ & $\begin{array}{c}\text { Expert } \\
\text { Opinion on } \\
\text { Drug Safety }\end{array}$ \\
\hline $\begin{array}{c}\text { Gourdy P et } \\
\text { al. }{ }^{26}\end{array}$ & $\begin{array}{c}\text { Hormonal contraception in } \\
\text { women at risk of vascular and } \\
\text { metabolic disorders: guidelines of } \\
\text { the French Society of } \\
\text { Endocrinology, } \\
\text { 2012. }\end{array}$ & $\begin{array}{c}\text { Annales } \\
\text { d'Endocrinolo } \\
\text { gie }\end{array}$ \\
& & \\
\hline
\end{tabular}

Figura 1: Número de publicações analisadas.

A DM vem atingindo patamares alarmantes e projeções preocupantes nos últimos anos. Estudos recentes mostram que a DM é um grave e crescente problema de saúde pública, tanto no Brasil quanto no mundo ${ }^{12}$, sendo que afetou 382 milhões de pessoas em todo o mundo em 2013, e este número deverá crescer para 592 milhões até $2035^{14}$. A Federação Internacional do Diabetes realizou um estudo em 2015 e contabilizou que 8,8\% (IC de 95\%, 7,2 a 11,4\%) da população mundial entre 20 e 79 anos de idade viviam com diabetes. No Brasil, o número de pessoas entre 20 e 79 anos de idade com diabetes em 2015 era de 14,3 (IC de 95\%, 12,9 a 15,8 ) milhões de pessoas, e em 2040 se projeta para 23,3 (IC de $95 \%, 21,0$ a 25,9) milhões de pessoas com esta patologia ${ }^{12}$.

A utilização de métodos contraceptivos também apresentou aumento expressivo nos últimos anos em todo mundo, saltando de 54,8\% (IC de 95\%, 52,3 a 57,1) nos anos 1990 , para $63,3 \%$ (IC de $95 \%, 60,4$ a 66,0 ) no ano de $2010^{15}$. No Brasil, apesar do alto índice de gestações na adolescência, em especial nos Estados do norte e nordeste ${ }^{16}, 65,2 \%$ das mulheres entre 15 e 49 anos de idade utilizam algum método moderno para prevenir gestações, sendo que este número passa para $67,8 \%$ ao incluir métodos tradicionais, como tabela e abstinência periódica ${ }^{15}$. Os contraceptivos hormonais são o método reversível mais utilizado pela população feminina brasileira ( $\pm 25 \%$ ) para planejamento familiar ${ }^{17}$.

A utilização de contraceptivo hormonal por pacientes diabéticas pode desencadear eventos adversos à saúde, dentre eles, os do sistema venoso são os mais perigosos e colocam a saúde desta paciente em risco. A trombose venosa profunda (TVP) é o principal e mais perigoso evento tromboembólico. Ela é desencadeada quando ocorre a formação de trombos dentro de veias profundas e as obstrui parcial ou totalmente, impedindo que seu fluxo siga normalmente. Ela ocorre geralmente nos membros inferiores ( 80 a $95 \%$ dos casos) e pode causar insuficiência venosa crônica (promovendo dor, edema, alterações na pigmentação da pele e úlceras) e embolia pulmonar (EP), que tem um alto índice de mortalidade devido sua gravidade. Aproximadamente 5 a 15\% dos indivíduos não tratados para TVP podem evoluir à óbito devido à EP. Os principais fatores diretamente ligados à gênese dos trombos são a estase sanguínea, a lesão endotelial e os estados de hipercoagulabilidade, portanto, idade avançada, câncer, procedimentos cirúrgicos, imobilização, uso de estrogênio, gravidez e distúrbios de hipercoagulabilidade hereditários ou adquiridos, constituem-se como fatores de risco para TVP. A 
sua incidência aumenta proporcionalmente com a idade, sugerindo que esta seja o fator de risco mais determinante para um primeiro evento de trombose ${ }^{18}$.

Tabela 2: Elegibilidade para uso de contracepção hormonal segundo a Organização Mundial da Saúde ${ }^{19}$.

Categoria Descrição Critérios

Categoria 1 método pode ser
utilizado sem qualquer

Histórico de câncer de

mama (atenção para

caso de mutação de

BRCA1); cirrose

compensada; hepatite

viral crônica.

Categoria 2 O método pode apresentar algum risco, provavelmente menor que o benefício. Ele pode ser usado, porém, com cautela. Deve haver acompanhamento clínico rigoroso.

\section{Categoria 3 Os riscos do método}

superam os benefícios,

e ele somente deve ser

utilizado se não houver

alternativa ou se a

paciente não aceitar

outro método. Ela deve

ser orientada quanto

aos riscos e deve ter

acompanhamento

médico rigoroso.
Tabagismo (menos de

35 anos de extensão);

diabetes mellitus sem

comprometimento de

órgãos (menos de 20

anos de extensão);

hipercolesteremia

(dependendo de outras

condições

cardiovasculares);

enxaqueca (menos de

35 anos de extensão);

hepatite viral (se a

paciente já utilizava

contraceptivo).

Tabagismo (mais de 35

anos de extensão e

menos de 15 cigarros

por dia); hipertensão

arterial sistêmica

controlada (pressão

arterial sistólica entre

140 e 159 mmHg e

pressão arterial

diastólica entre 90 e 99

$\mathrm{mmHg}$ ); enxaqueca

(mais de 35 anos de

extensão); múltiplos

fatores de risco

cardiovascular; câncer de mama (pós 5 anos);

lactação (pós 6

semanas).

Categoria 4 O uso do método

Tabagismo (mais de 35

representa um risco

anos e mais de 15

inaceitável à saúde, não devendo ser utilizado

cigarros por dia);

hipertensão arterial pela paciente.

sistêmica não

controlada (pressão

arterial sistólica maior

de $60 \mathrm{mmHg}$ e pressão

arterial diastólica maior

de $100 \mathrm{mmHg}$ );

histórico de cardiopatia isquêmica, trombose

venosa profunda e

embolia pulmonar;

hipercoagulabilidade;

enxaqueca com aura;

câncer de mama;

lactação (antes de 6

semanas); cirrose

descompensada;

hepatite aguda.

Estudos recentes, com o objetivo de mostrar a importância da prescrição mais cautelosa de anticoncepcionais e do monitoramento de pacientes em idade reprodutiva com diabetes mellitus, mostram a importância do tema em questão. A Organização Mundial da Saúde (OMS) estabeleceu critérios norteadores para prescrição de contracepção hormonal, que visa orientar os profissionais da saúde quanto à prescrição de contraceptivos, classificando de acordo com a saúde da pretendente ao método (se ela apresenta alguma condição especial que limite ou impeça o uso do contraceptivo), dentre elas, a DM ${ }^{19}$. A Tabela 2 mostra a estratificação. Caso a paciente não apresentar nenhum outro fator de risco associado ao diabetes e mantiver os indicies glicêmicos sob controle, ela se enquadra na categoria 2. Nestas condições estará liberada para optar por qualquer um dos métodos, desde que tenha acompanhamento médico. Se a paciente com diabetes apresentar complicações mais graves, como retinopatias, nefropatias, neuropatia, e diabetes há mais de 20 anos, ela se enquadra na categoria 3 ou 4 (dependendo da gravidade) e não estará liberada para utilizar contraceptivos considerados de alto risco (AOC, anel vaginal e depósito de drosperidona), apenas anticoncepcionais considerados seguros, como implantes subdérmicos, DIU (de levonogestrel ou cobre), pílulas só de progestogênio ou, em alguns casos, métodos de barreiras ${ }^{17,19,20,21}$. 
O’Brien et al. ${ }^{22}$ mostrou que há segurança no uso de contracepção hormonal em mulheres com diabetes tipo 1 e tipo 2, pois encontrou baixo risco absoluto, de aproximadamente 1 evento tromboembólico por 100 mulheres/ano de uso. Os contraceptivos hormonais combinados, que contêm estrogênio e progesterona, aumentam o risco de tromboembolismo na população geral e também nas mulheres com DM tipo 1 e tipo 2; já que o estrogênio exerce influência negativa na via da proteína $C$ e é provavelmente o mecanismo primário para o efeito prótrombótico dos contraceptivos hormonais combinados, bem como dos hormônios pós-menopausa ${ }^{22}$. A utilização de contracepção hormonal combinada apresenta a mesma probabilidade de causar eventos tromboembólicos em mulheres com e sem diabetes, mas o risco absoluto em mulheres com diabetes é maior devido à doença, que é um agravante para estes incidentes ${ }^{23}$. $\mathrm{O}^{\prime}$ Brien et al. ${ }^{22}$ relata ainda, que mulheres diabéticas que utilizam contracepção com estrogênio têm risco maior de desenvolver complicações trombóticas quando comparadas às que utilizam progestogênio ou as não usuárias. Os métodos anticoncepcionais mais associados aos eventos tromboembólicos para mulheres com DM são os adesivos e o anel vaginal, ao passo que os métodos hormonais com menor probabilidade para incidência de eventos tromboembólicos são os implantes subdérmicos e o dispositivo intrauterino, que são até 20 vezes mais eficazes que os contraceptivos orais ${ }^{22}$.

O ganho de peso associado ao uso de métodos anticoncepcionais hormonais é uma importante razão para a descontinuação do método, pois é o argumento de muitas mulheres que abandonam $o$ uso de métodos anticoncepcionais ${ }^{24}$. O aumento de peso é um dado importante quando se trata de pacientes diabéticas, uma vez que a obesidade se destaca como um dos principais fatores de risco para o desenvolvimento de DM tipo ${ }^{12}$. Não há evidência, contudo, de que o uso de anticoncepcional oral hormonal cause ganho de peso; excentricamente, um estudo aponta ganho de peso ao final de cinco anos em mulheres que utilizam acetato de medroxiprogesterona de depósito quando comparadas às que fazem uso de sistema intrauterino liberador de $20 \mu \mathrm{g} / \mathrm{dia}$ de levonorgestrel ou as usuárias de dispositivo intrauterino $T$ cobre $380 \mathrm{~A}^{24}$. Ademais, contraceptivos orais apenas de progestágeno ou acetato de medroxiprogesterona de depósito injetável, produzem até quatro vezes mais chances de desencadear eventos tromboembólicos quando comparados ao DIU 22 .

Shufelt et al. ${ }^{25}$ demonstraram preocupação quanto aos acidentes cardiovasculares associados à utilização de contraceptivos orais. Segundo eles, uma variedade de dados básicos, animais e humanos sugerem que os hormônios contraceptivos têm efeitos anti-ateroscleróticos. Há associação positiva entre história de ciclos menstruais irregulares e eventos cardiovasculares adversos, sugerindo que a disfunção ovulatória e os níveis de estrogênio relativamente baixos também podem elevar o risco cardiovascular. Os hormônios contraceptivos suprimem os andrógenos ovarianos e aumentam a globulina de ligação aos hormônios sexuais, reduzindo assim, a fração livre da testosterona plasmática. Este é um mecanismo útil de ação destes fármacos em mulheres com síndrome dos ovários policísticos, uma condição que pode estar associada a um risco cardiovascular elevado. As formulações contraceptivas orais atualmente em uso não indicam aumento do risco de IAM, mas um aumento persistente do risco de tromboembolismo venoso. Mulheres com 35 anos ou mais devem ser avaliadas quanto ao risco cardiovascular, incluindo hipertensão, tabagismo, diabetes, nefropatia e outras doenças vasculares antes da prescrição para o uso de contraceptivos orais, uma vez que o estrogênio tem conhecido efeito pró-trombótico e, por tal, eleva o tromboembolismo venoso cardiovascular ${ }^{25}$. Em mulheres com DM, a trombose arterial é mais comum quando comparada à venosa 22

Alguns estudos sugerem a interferência de estrógeno e progestágeno sobre o metabolismo de carboidratos, todavia, de modo geral, a grande maioria dos estudos epidemiológicos sugerem que os contraceptivos orais não aumentam o risco de diabetes ${ }^{26}$. Evidências sugerem que não há grandes diferenças no metabolismo de carboidratos entre os diferentes contraceptivos hormonais em mulheres sem diabetes ${ }^{27}$.

Uma situação específica também merece atenção: mulheres com histórico de diabetes gestacional. Gravidez com complicações devido à diabete gestacional ocorrem entre 0,6 e $0,9 \%$ de todas as gestações e estão associadas à hiperglicemia materna que se mostra de difícil manejo devido às rápidas condições fisiológicas que proporcionam alterações na sensibilidade da insulina durante o curso da gestação, necessitando de acompanhamento rigoroso ${ }^{28}$. Segundo Gourdy et al. $^{26}$, estudos de contracepção neste grupo específico são raros e, os resultados apresentados à luz da literatura atual, dão conta que a contracepção baseada em combinações estrogênio e progestina, progesterona isolada, implante de levonorgestrel ou um DIU (com ou sem progestina) pode ser usado normalmente em pacientes com história de diabetes gestacional. Na ausência de contraindicações significativas de risco venoso ou arterial, a combinação de estrogênio e progestina continua sendo uma excelente escolha para mulheres que optam pela contracepção hormonal ${ }^{26}$.

Para Lidegaard ${ }^{29}$, a primeira escolha em mulheres com menos de 35 anos deve ser uma pílula combinada de baixo risco, ou seja, com um progestogênio de segunda geração, com a dose mais baixa de estrogênio. Mulheres jovens com fatores de risco de trombose como idade acima de 35 anos, predisposições genéticas, adiposidade, síndrome dos ovários policísticos, diabetes, tabagismo, hipertensão ou enxaqueca com aura não devem usar produtos de alto risco, mas devem considerar principalmente produtos com apenas progestágeno e tenha cuidado ao usar produtos combinados de baixo risco ${ }^{29}$. Em mulheres com diabetes tipo I, a contracepção de associação com estrogênio e progesterona pode ser proposta se não houver fatores de risco vasculares ou complicações angiopáticas; a contracepção somente com progestogênio e DIU são alternativas no caso de complicações documentadas (nefropatia proteinúrica ou insuficiência renal, retinopatia avançada, neuropatia ou doença cardiovascular). Em mulheres com diabetes tipo II, a prescrição de anticoncepcionais estrogênicos e progestínicos deve ser restrita às seguintes circunstâncias: ausência de obesidade (com índice de massa corporal abaixo de $30 \mathrm{~kg} / \mathrm{m}^{2}$ ), ausência de fatores de risco cardiovascular e ausência de complicações angiopáticas; em todos os outros casos, a contracepção hormonal deve basear- 
se apenas numa progestina, independentemente do modo de administração ${ }^{26}$. Para o AVC trombótico, cada um dos fatores de risco praticamente dobra o risco, enquanto que para o IAM, o tabagismo implica um risco relativo de quatro, o tabagismo severo e a diabetes, um risco relativo de $\operatorname{cinco}^{29}$. Uma questão importante é o receio de que a contracepção hormonal possa agravar as complicações diabéticas, incluindo os distúrbios microvasculares. As complicações microvasculares, no entanto, não são mais frequentes nem mais graves em pacientes com DM tipo I que usam um AOC: vários estudos não mostraram efeito deletério ${ }^{26}$.

Uma pesquisa realizada no nordeste do Brasil buscou analisar o conhecimento de mulheres com DM em relação aos métodos contraceptivos ${ }^{8}$. Foram entrevistadas mulheres com DM tipo I e tipo II em idade reprodutiva (18-49 anos) e, lamentavelmente, quase $71 \%$ das sujeitas foram classificadas como possuindo "nenhum conhecimento" sobre os métodos anticoncepcionais, 26\% apresentaram "conhecimento limitado", 3\% apresentaram "conhecimento moderado" e nenhuma entrevistada foi categorizada com "conhecimento substancial" ou "extenso". Chama a atenção o dado que 53\% das participantes da pesquisa fazem uso de métodos contraceptivos por conta própria, sem um auxílio especializado, mesmo sabendo que $37 \%$ delas possuem sobrepeso ou obesidade $^{8}$, fator de risco importante no tangente à escolha correta de método. Estes dados revelam a importância de se aumentar o conhecimento sobre contracepção, uma vez que o esclarecimento e o conhecimento empoderam os indivíduos e os tornam mais lúcidos para escolhas mais adequadas e saudáveis. Obstetras e ginecologistas frequentemente estão em posição de aconselhar as mulheres sobre suas opções contraceptivas com potencial para diminuir o número de gravidezes indesejadas e reduzir o número de abortos; além disso, para reduzir a taxa de aborto repetido ${ }^{30}$. O resultado de políticas públicas de disseminação do conhecimento é, inexoravelmente, a perspectiva de uma população mais saudável e apta a fazer escolhas inteligentes na seara da saúde. Esta atitude, apesar de demandar longos prazos, desonera o montante gasto pelos cofres públicos no alento das mazelas que açoitam 0 indivíduo ignorante. Melhorando o conhecimento das pacientes, tanto das que sofrem com diabetes tanto das que não possuem a doença, sua qualidade de vida melhora e são minimizados os efeitos da contracepção inadequada e de gestações não planejadas.

Tanto a DM do tipo I quanto a do tipo II são, portanto, grave e crescente problema de saúde pública, que deve atingir números alarmantes nas próximas décadas, tanto no Brasil quanto no mundo ${ }^{12}$. Juntamente com a DM, houve um aumento no uso da contracepção hormonal ${ }^{15}$ que, que combinado com a DM, pode aumentar a incidência de eventos tromboembólicos. É necessário um cuidado maior na prescrição de contracepção hormonal para este grupo de pacientes, pois a DM em si, já é considerado um fator de risco para incidentes venosos e arteriais ${ }^{18}$. Deve-se dar prioridade para métodos que tenha uma elevada eficácia na prevenção de gestações e baixo risco de desencadear eventos adversos à saúde destas pacientes. A escassez de estudos relacionados à população feminina diabética em idade reprodutiva e que utilizam métodos contraceptivos hormonais no Brasil, mostra a importância de se realizar mais estudos. Buscar mais segurança e qualidade na prescrição de métodos anticoncepcionais para estas mulheres seguindo os critérios de elegibilidade norteados pela ciência é de suma importância para uma contracepção segura e eficaz. Apesar da crescente variedade de métodos contraceptivos, o manejo do controle de riscos relacionados ao uso de anticoncepcionais hormonais é uma questão complexa, mas crucial. É importante ressaltar que os fatores de risco vasculares raramente se apresentam isoladamente, e que a existência de vários fatores de risco impõe ainda maior cautela na prescrição desse tipo de combinação hormonal ${ }^{26}$. Finalmente, este documento é pretencioso em alertar a comunidade científica quanto aos meandros impostos pelo uso irracional de contraceptivos hormonais pela crescente população diabética. A pluralidade farmacológica (e metodológica) disposta no mercado atual aliado ao apoio de profissional esperto, marcam a chave para o desfecho adequado deste certame.

\section{REFERÊNCIAS}

1. Giglio MRP, Melo GP, Ferreira VG, Albernaz MA, Ribeiro MO. Conhecimentos dos médicos residentes de ginecologia $e$ obstetrícia sobre contracepção hormonal em situações especiais. Revista Brasileira de Educação Médica 2017;41(1):69-78.

2. Passos EP, Ramos JGL, Martins-Costa SH, Magalhães JA, Menke CH, Freitas F. Rotinas em Ginecologia. 7ạ edição. Porto Alegre: Artmed, 2017.

3. Hurt JK, Guile MW, Bienstock JL, Fox HE, Wallach EE. Manual de ginecologia e obstetrícia do Johns Hopkins. 4ạ edição. Porto Alegre: Artmed, 2012.

4. Monteiro AA. Novos métodos contraceptivos. Revista Portuguesa de Medicina Geral e Familiar 2005;21(5):475-483.

5. Veloso DLC, Peres VC, Lopes JSOC, Salge AKM, Guimarães JV. Anticoncepção de emergência: conhecimento e atitude de acadêmicos de enfermagem. Revista Gaúcha de Enfermagem 2014;35(2):33-39.

6. Vieira CA, Brito MB, Yazlle MEHD. Contracepção no puerpério. Revista Brasileira de Ginecologia e Obstetrícia 2008;30(9):470-479.

7. Machado RR, Monteiro IMU, Magalhães J, Guazzelli CAF, Brito MB, Finotti MF, Lubianca JN, Sakamoto LC, Franceschini SA. Long-acting reversible contraception. Revista Brasileira de Ginecologia e Obstetrícia 2017;39(6):294-308.

8. Evangelista DR, Moura ERF, Costa CBJS, Bezerra CG, Valente MMQP, Sousa CSP. Conhecimento e prática anticoncepcional de mulheres portadoras de Diabetes Mellitus. Escola Anna Nery 2014;18(3):441-447.

9. Mello KF, Lunardelli A, Donadio MVF, Caberlon E, Oliveira CSA, Bastos CMA, Pires MGS, Nunes FB, Oliveira JR. Immunomodulatory effects of oral antidiabetic drugs in lymphocyte cultures from patients with type 2 diabetes. Jornal Brasileiro de Patologia e Medicina Laboratorial 2011;47(1):41-46.

10. Mazzaferro GS, Lunardelli A. Frutosamina como principal parâmetro glicêmico do paciente diabético em hemodiálise. Ciência \& Saúde 2016;9(2):119-126.

11. Neves RC, Duro SMS, Muñiz J, Castro TRP, Facchini LA, Tomasi E. Estrutura das unidades básicas de saúde para atenção às pessoas com diabetes: ciclos I e II do Programa Nacional de Melhoria do Acesso e da Qualidade. Cadernos de Saúde Pública 2018;34(4):110.

12. Diretrizes da Sociedade Brasileira de Diabetes 2017-2018. São Paulo: Clannad, 2017.

13. Skouby SO. Hormonal contraception in obesity, the metabolic syndrome, and diabetes. Annals of the New York Academy of Sciences 2010;1205:240-244. 
14. Urakami T, Kuwabara R, Yoshida K. Economic Impact of Diabetes in Japan. Current Diabetes Reports 2019;1.

15. Farias MR, Leite SN, Tavares NUL, Oliveira MA, Arrais PSD, Bertoldi AD, Pizzol TSD, Luiza VL, Ramos LR, Mengue SS. Utilização e acesso a contraceptivos orais e injetáveis no Brasil. Revista de Saúde Pública 2016;50(supl 2):1-10.

16. Sousa MMCR, Gomes KRO. Conhecimento objetivo e percebido sobre contraceptivos hormonais orais entre adolescentes com antecedentes gestacionais. Cadernos de Saúde Pública 2009;25(3):645-654.

17. Brito MB, Nobre F, Vieira CSV. Contracepção hormonal e sistema cardiovascular. Arquivos Brasileiros de Cardiologia 2011;96(4):8189.

18. Presti C, Miranda F, Pânico MDB, Matielo MF, Porto CLL, Marques MA, Yoshida RA. Trombose venosa profunda - diagnóstico e tratamento. Diretrizes da Sociedade Brasileira de Angiologia e de Cirurgia Vascular, 2015.

19. World Health Organization. Medical eligibility criteria for contraceptive use - part II. 5a edição. Genebra: WHO Library Cataloguing, 2015.

20. Federação Brasileira das Associações de Ginecologia e Obstetrícia. Critérios médicos de elegibilidade da OMS para uso de métodos anticoncepcionais. FEBRASGO, 2010.

21. BRASIL, Ministério da Saúde. Uso racional de medicamentos: temas selecionados. Brasília: Ministério da Saúde, 2012.

22. O'Brien SH, Koch T, Vesely SK, Schwarz EB. Hormonal contraception and risk of thromboembolism in women with diabetes. Diabetes Care 2017;40(2):233-238.

23. Skouby SO. Hormonal contraception in obesity, the metabolic syndrome, and diabetes. Annals of the New York Academy of Sciences 2010;1205(1):240-244.

24. Yela DA, Monteiro IMU, Bahamondes LG, Castillo S, Bahamondes MV, Fernandes A. Variação de peso em usuárias de sistema intrauterino liberador de levonorgestrel, DIU T-cobre e acetato de medroxiprogesterona no Brasil. Revista da Associação Médica Brasileira 2006;52(1):32-36.

25. Shufelt CL, Bairey Merz CN. Contraceptive hormone use and cardiovascular disease. Journal of the American College of Cardiology 2009;53(3):221-231.

26. Gourdy P, Bachelot A, Catteau-Jonard S, Chabbert-Buffet N, Christin-Maître S, Conard J, Fredenrich A, Gompelh A, LamicheLorenzini $F$, Moreaui $C$, Plu-Bureau G, Vambergue A, Vergès $B$, Kerlan. Hormonal contraception in women at risk of vascular and metabolic disorders: guidelines of the French Society of Endocrinology. Annales d'Endocrinologie 2012;73:469-487.

27. Lopez LM, Grimes DA, Schulz KF. Steroidal contraceptives: effect on carbohydrate metabolism in women without diabetes mellitus. Cochrane Database of Systematic Reviews 2014;(4):CD006133.

28. Hauffe F, Schaefer-Graf UM, Fauzan R, Schohe AL, Scholle $D$, Sedlacek L, Scherer KA, Klapp C, Ramsauer B, Henrich W, Schlembach D, Abou-Dakn M. Higher rates of large-forgestational-age newborns mediated by excess maternal weight gain in pregnancies with type 1 diabetes and use of continuous subcutaneous insulin infusion vs multiple dose insulin injection. Diabetic Medicine 2019;36(2):158-166.

29. Lidegaard $\varnothing$. Hormonal contraception, thrombosis and age. Expert Opinion on Drug Safety 2014;13(10):1353-1360.

30. Querido ACCM, Silveira DF, Lima NA, Nunes MMF, Venturoso ICCM. Uso de contraceptivos de longa duração: uma revisão de literatura. Revista de Patologia do Tocantins 2018;5(4):61-63. 